\title{
Cyclic AMP Suppresses the Inhibition of Glycolysis by Alternative Oxidizable Substrates in the Heart
}

\author{
Christophe Depre, Sylvie Ponchaut, Johan Deprez, Liliane Maisin, and Louis Hue \\ Hormone and Metabolic Research Unit, International Institute of Cellular and Molecular Pathology, and Division of Cardiology, Louvain \\ University Medical School, Brussels, Belgium
}

\begin{abstract}
In normoxic conditions, myocardial glucose utilization is inhibited when alternative oxidizable substrates are available. In this work we show that this inhibition is relieved in the presence of CAMP, and we studied the mechanism of this effect. Working rat hearts were perfused with $5.5 \mathrm{mM}$ glucose alone (controls) or together with $5 \mathrm{mM}$ lactate, $5 \mathrm{mM}$ $\beta$-hydroxybutyrate, or $1 \mathrm{mM}$ palmitate. The effects of $0.1 \mathrm{mM}$ chlorophenylthio-cAMP (CPT-cAMP), a cAMP analogue, were studied in each group. Glucose uptake, flux through 6-phosphofructo-1-kinase, and pyruvate dehydrogenase activity were inhibited in hearts perfused with alternative substrates, and addition of CPT-cAMP completely relieved the inhibition. The mechanism by which CPT-cAMP induced a preferential utilization of glucose was related to an increased glucose uptake and glycolysis, and to an activation of phosphorylase, pyruvate dehydrogenase, and 6-phosphofructo2-kinase, the enzyme responsible for the synthesis of fructose 2,6-bisphosphate, the well-known stimulator of 6-phosphofructo-1-kinase. In vitro phosphorylation of 6-phosphofructo-2-kinase by cAMP-dependent protein kinase increased the $V_{\max }$ of the enzyme and decreased its sensitivity to the inhibitor citrate. Therefore, in hearts perfused with various oxidizable substrates, cAMP induces a preferential utilization of glucose by a concerted stimulation of glucose transport, glycolysis, glycogen breakdown, and glucose oxidation. (J. Clin. Invest. 1998. 101:390-397.) Key words: cyclic AMP • glucose $\bullet$ fructose 2,6-bisphosphate - lactate • working heart
\end{abstract}

\section{Introduction}

Chemical energy for the working heart is provided by the oxidation of various substrates, such as fatty acids, ketone bodies, and carbohydrates. In normoxic conditions, glucose is not a preferred substrate for the heart, because its metabolism is inhibited by fatty acids and ketone bodies $(1,2)$. This glucosesparing effect, also known as the "glucose/fatty acid cycle," results from the inhibition of pyruvate dehydrogenase (PDH),

Address correspondence to Prof. L. Hue, HORM Unit, ICP-UCL 7529, Avenue Hippocrate, 75, B-1200 Brussels, Belgium. Phone: 32-2764-75-29; FAX: 32-2-762-74-55; E-mail: hue@horm.ucl.ac.be

Received for publication 11 July 1997 and accepted in revised form 10 November 1997.

J. Clin. Invest.

(C) The American Society for Clinical Investigation, Inc. 0021-9738/98/01/0390/08 \$2.00

Volume 101, Number 2, January 1998, 390-397

http://www.jci.org 6-phosphofructo-1-kinase (PFK-1), and glucose transport (3-6). Lactate also inhibits glycolysis in what could be regarded as an extension of the glucose/fatty acid cycle $(7,8)$.

Besides substrate availability, heart metabolism is also regulated by hormones, some of them acting through cyclic nucleotides. cAMP is a mediator of epinephrine, thyroid hormones, glucagon, histamine, or prostacyclin in the heart (9-11), while cGMP is the intracellular messenger of atrial natriuretic peptide and nitric oxide $(12,13)$. It is well known that addition of epinephrine to isolated perfused hearts stimulates both cardiac work and glucose utilization $(14,15)$, suggesting that the extra energy required for the increased mechanical activity is provided by glucose. However, it is not known whether these effects are mediated only through cAMP production. Moreover, it is still unclear by which mechanism cAMP may relieve the glycolytic inhibition exerted by alternative fuels. Our aim was to answer these questions. Namely, we sought to delineate the effects of a permeable cAMP analogue on glucose metabolism in the working rat heart and to determine whether, and by which mechanism, the cAMP-signaling pathway may relieve inhibition of glucose utilization exerted by alternative oxidizable substrates.

\section{Methods}

Perfusion protocol. Male Wistar rats (170-200 g) fed ad libitum were anesthetized with pentobarbital $(50 \mathrm{mg} / \mathrm{kg}$, intraperitoneally). The heart was quickly removed and perfused under working conditions at $37^{\circ} \mathrm{C}$ for the indicated periods of time in a recirculation system with $100 \mathrm{ml}$ of a Krebs-Henseleit bicarbonate buffer containing $5.5 \mathrm{mM}$ glucose (16) and in equilibrium with a gas phase containing $95 \% \mathrm{O}_{2}$ and $5 \% \mathrm{CO}_{2}$. Two working conditions were chosen, a low load condition, in which the preload and the afterload were 10 and $60 \mathrm{~cm}$ water, respectively, and a high load condition, in which the preload and the afterload were increased to 15 and $120 \mathrm{~cm}$ water, respectively. When indicated, the hearts were perfused with $5.5 \mathrm{mM}$ glucose together with $5 \mathrm{mM}$ lactate, $5 \mathrm{mM} \beta$-hydroxybutyrate, or $1 \mathrm{mM}$ palmitate with $2 \%$ (wt/vol) defatted albumin (Boehringer Mannheim, Mannheim, Germany). The effects of $0.1 \mathrm{mM}$ 8-chlorophenylthio-cAMP (CPTcAMP; Sigma Chemical Co., St. Louis, MO), a nondegradable cAMP analogue, were studied in each group.

Heart rate, aortic pressures, and aortic and coronary outputs were regularly measured. The work produced by the heart is expressed by unit of time as a hydraulic power (grams · meter/minute) $(16,17)$.

Analytical methods. Glucose phosphorylation was assessed by the rate of detritiation of $\left[2-{ }^{3} \mathrm{H}\right]$ glucose (18). The flux through PFK-1 from exogenous glucose was estimated by the rate of detritiation of $\left[3-{ }^{3} \mathrm{H}\right]$ glucose (19). Tracer amounts of glucose $(2 \mu \mathrm{Ci} / 100 \mathrm{ml}$ perfu-

1. Abbreviations used in this paper: CPT-cAMP, chlorophenylthiocAMP; Fru-6-P, fructose 6-phosphate; Fru-1,6-P2, fructose 1,6-bisphosphate; Fru-2,6-P2, fructose 2,6-bisphosphate; PDH, pyruvate dehydrogenase; PFK-1, 6-phosphofructo-1-kinase; PFK-2, 6-phosphofructo-2-kinase; PKA, cAMP-dependent protein kinase. 
sate) were added to the buffer at the beginning of the working perfusion. Samples were removed periodically from the perfusate to measure the formation of ${ }^{3} \mathrm{H}_{2} \mathrm{O}$. Separation of radioactive water from labeled glucose was carried out by column chromatography, as described (18). The results are expressed as micromoles of glucose detritiated per minute per gram. The detritiation rates are minimal values because they may somewhat underestimate the true rates. This may result from incomplete detritiation (usually negligible in muscle tissue) and it may also occur when the intracellular hexose-phosphate pool is diluted by unlabeled glucosyl units coming from glycogen (19, 20). Taking into account the concentrations of hexose-phosphates measured in our experimental conditions, we calculated that the underestimation amounted to $<10 \%$.

At the end of perfusion, the hearts were freeze-clamped between aluminum blocks precooled in liquid nitrogen. About $300 \mathrm{mg}$ frozen tissue was homogenized with an Ultraturrax in 3 vol of $10 \%$ perchloric acid at $0-4^{\circ} \mathrm{C}$ to measure glycolytic intermediates (glucose 6-phosphate and fructose 6-phosphate [Fru-6-P] referred to as hexose 6-phosphates and fructose 1,6-bisphosphate [Fru-1,6-P2], respectively) and citrate (21). Fructose 2,6-bisphosphate (Fru-2,6-P2) was measured in alkaline extracts, as described previously (5). Glycogen was measured enzymatically and expressed as glucose equivalents (22). When the hearts were ready to be perfused in working condition (zero time of perfusion), their glycogen content averaged $14 \pm 3 \mu \mathrm{mol} / \mathrm{g}$ compared with $\sim 20 \mu \mathrm{mol} / \mathrm{g}$ in hearts in vivo, thus indicating a loss of $\sim 6 \mu \mathrm{mol} / \mathrm{g}$ during the preparation of the experiment.

Oxygen consumption was measured by a Clark electrode (Yellow Spring, $\mathrm{OH}$ ) connected to the pulmonary artery by a short thick-wall Tygon tube.

Measurement of enzyme activities. The activity of phosphorylase was measured at $30^{\circ} \mathrm{C}$ in the presence of $5 \mathrm{mM}$ caffeine (active form) or $2 \mathrm{mM}$ AMP (total activity) as described (22) in homogenates prepared from frozen heart tissue (23).

To measure PDH activity, frozen samples $(100 \mathrm{mg})$ of ventricles were homogenized in $9 \mathrm{vol}$ of a buffer $(0.1 \mathrm{M}$ potassium phosphate, $2 \mathrm{mM}$ EDTA, $1 \mathrm{mM}$ dithiothreitol, $\mathrm{pH}$ 7.3) containing $0.1 \%$ (wt/vol) Triton X-100 and $50 \mu \mathrm{l} / \mathrm{ml}$ fresh rat serum, and then frozen at $-20^{\circ} \mathrm{C}$. After thawing, the homogenates were centrifuged (Eppendorf, $30 \mathrm{~s}$ ) and the active form in the supernatant was assayed immediately at $30^{\circ} \mathrm{C}$, using a coupling reaction with arylamine acetyltransferase as described (24). The proportion of PDH in the active form was expressed as a percentage of the total activity, which was determined following incubation of the supernatant with purified pig heart phosphatase and in the presence of $1 \mathrm{mM} \mathrm{Ca}^{2+}$ and $25 \mathrm{mM} \mathrm{Mg}^{2+}(25)$.

Proteins were measured with bovine serum albumin as a standard. $1 \mathrm{U}$ of enzyme activity corresponds to the formation of $1 \mu \mathrm{mol}$ of product/min under the stated conditions.

In vitro phosphorylation of 6-phosphofructo-2-kinase (PFK-2). Bovine heart PFK-2/FBPase-2 was purified and phosphorylated by the purified catalytic subunit of cAMP-dependent protein kinase (PKA) as described (26). The conditions used to study the effects of phosphorylation on PFK-2 inhibition by citrate are detailed in the appropriate legend.

Statistical analysis and expression of results. All data are expressed per gram of wet weight and are the means \pm standard error for at least five different hearts. Unpaired two-tailed Student's $t$ test was used to evaluate the statistical significance of differences. ANOVA with Bonferroni correction was performed when necessary. A value of $P<$ 0.05 was considered as statistically significant.

\section{Results}

\section{Inhibition of glycolysis by alternative substrates}

Two work load conditions were tested to assess the workdependent stimulation of glucose metabolism in hearts perfused with glucose alone or together with another substrate. In hearts perfused with $5.5 \mathrm{mM}$ glucose alone, increasing the work load doubled hydraulic power and glycolysis from exogenous glucose (Fig. 1). In agreement with a previous study (16), it also increased the concentration of Fru-2,6-P2, decreased hexose 6-phosphate concentration, and did not affect Fru-1,6$\mathrm{P} 2$, glycogen, and citrate concentration (Table I). Addition of lactate, $\beta$-hydroxybutyrate, or palmitate together with glucose did not affect hydraulic power (Fig. 1). In keeping with Randle's original observation (2), these substrates totally suppressed the stimulation of the flux through PFK-1 from exoge-
Low Load
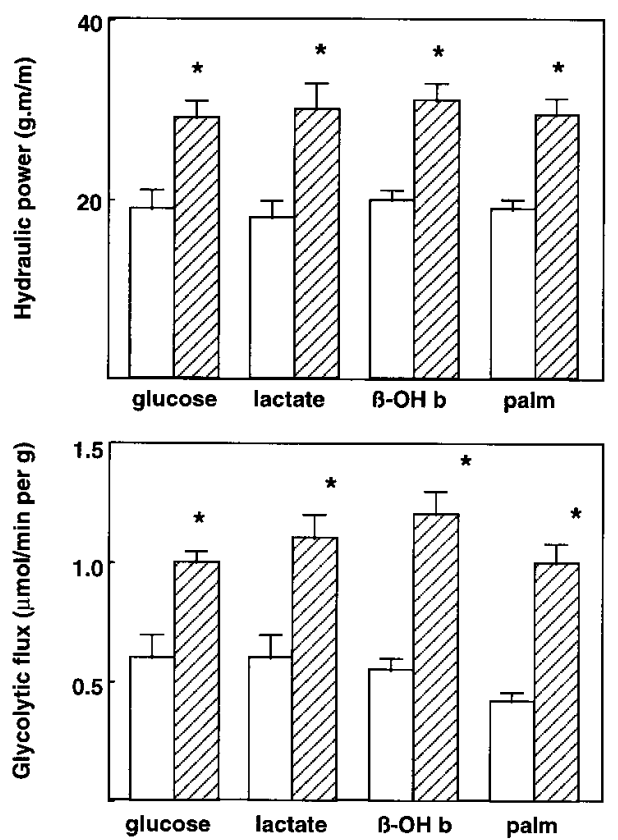

High Load
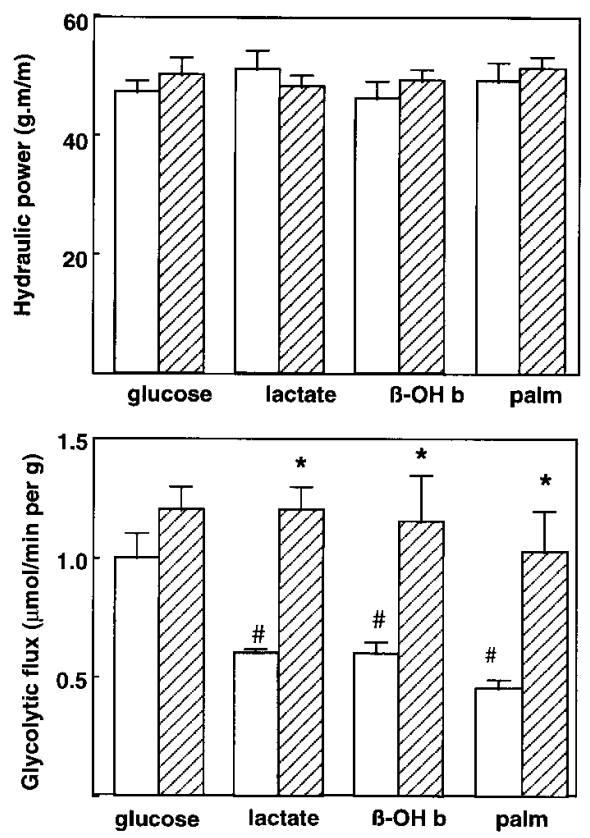

Figure 1. Hydraulic power (top) and glycolytic flux (bottom) in hearts perfused with $5.5 \mathrm{mM}$ glucose alone or together with $5 \mathrm{mM}$ lactate, $5 \mathrm{mM} \beta$-hydroxybutyrate, or $1 \mathrm{mM}$ palmitate, at either low load (left) or high load $(r i g h t)$. Glycolytic flux represents the rate of detritiation of $\left[3-{ }^{3} \mathrm{H}\right]$ glucose. Open bars, control hearts; hatched bars, hearts perfused with $0.1 \mathrm{mM}$ CPT-cAMP. $* P<0.05$ vs. same group without CPT-cAMP; ${ }^{\#} P<0.05$ vs. hearts with glucose alone in same condition; $n \geq 6$ in each group. 
Table I. Effect of CPT-cAMP on the Concentration of Glycogen, Hexose 6-P, Fru-1,6-P2, Citrate, and Fru-2,6-P2 in Hearts Perfused during 20 min in Two Work Load Conditions, with Glucose (glu) or Glucose and Lactate (glu/lac)

\begin{tabular}{|c|c|c|c|c|c|c|c|}
\hline Work load & Substrate & CPT-cAMP & Glycogen & Hexose 6-P & Fru-1,6-P2 & Citrate & Fru-2,6-P2 \\
\hline & & & $\mu \mathrm{mol} / \mathrm{g}$ & & $\mathrm{nmol} / \mathrm{g}$ & & \\
\hline \multirow[t]{4}{*}{ Low load } & glu & - & $10 \pm 1$ & $84 \pm 10$ & $26 \pm 4$ & $80 \pm 10$ & $2.1 \pm 0.2$ \\
\hline & & + & $1 \pm 0.1^{*}$ & $80 \pm 6$ & $40 \pm 6$ & $27 \pm 5^{*}$ & $3.0 \pm 0.2 *$ \\
\hline & glu/lac & - & $16 \pm 2^{\ddagger}$ & $195 \pm 20^{\ddagger}$ & $42 \pm 5$ & $160 \pm 15^{\ddagger}$ & $0.8 \pm 0.2^{\ddagger}$ \\
\hline & & + & $3 \pm 0.5^{*}$ & $215 \pm 25^{*}$ & $72 \pm 6^{*}$ & $135 \pm 20$ & $2.5 \pm 0.3 *$ \\
\hline \multirow[t]{4}{*}{ High load } & glu & - & $11 \pm 2$ & $52 \pm 5$ & $40 \pm 5$ & $85 \pm 2$ & $3.0 \pm 0.2^{\S}$ \\
\hline & & + & $0.2 \pm 0.1 * \S$ & $65 \pm 10$ & $33 \pm 4$ & $20 \pm 5^{*}$ & $2.8 \pm 0.2$ \\
\hline & glu/lac & - & $15 \pm 2^{\ddagger}$ & $185 \pm 12^{\ddagger}$ & $51 \pm 3$ & $125 \pm 15^{\ddagger}$ & $1.0 \pm 0.3^{\ddagger}$ \\
\hline & & + & $2 \pm 0.2 * \S$ & $88 \pm 8 * \S$ & $80 \pm 5^{*}$ & $70 \pm 10 *$ & $2.5 \pm 0.3^{*}$ \\
\hline
\end{tabular}

Hearts were freeze-clamped after 20 min of perfusion in each condition. Hexose 6- $P$, hexose 6-phosphates. The values are the means \pm SEM for at least five different perfusions. ${ }^{*} P<0.05$ vs. corresponding value without CPT-cAMP. ${ }^{\ddagger} P<0.05$ vs. corresponding value in the same work load condition with glucose alone. ${ }^{\S} P<0.05$ vs. corresponding value at low load.
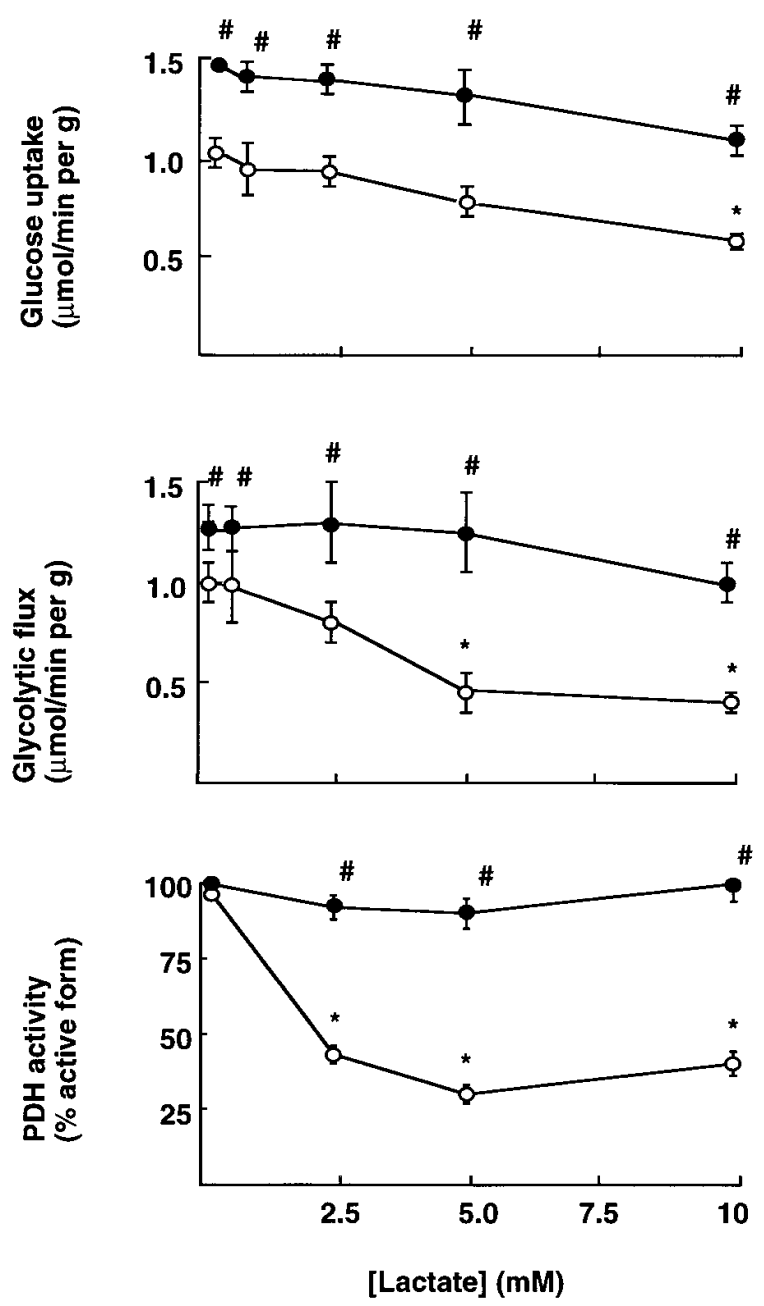

Figure 2. Glucose uptake (top), glycolytic flux (middle), and PDH activity (bottom) in hearts perfused at high load with various concentrations of lactate without (open symbols) or with (closed symbols) $0.1 \mathrm{mM}$ CPT-cAMP. Glucose uptake and glycolytic flux represent the rate of detritiation of $\left[2-{ }^{3} \mathrm{H}\right]$ and $\left[3-{ }^{3} \mathrm{H}\right]$ glucose, respectively. $* P<$ 0.05 vs. same group in the absence of lactate; ${ }^{\#} P<0.05$ vs. corresponding value in the absence of CPT-cAMP. $n \geq 6$ in each group. nous glucose, which was induced by increasing the work load (Fig. 1). Therefore, heart work by itself did not stimulate glucose metabolism when other oxidizable substrates were available.

The inhibitory effects of lactate on glucose metabolism were further studied. Perfusion with lactate within the physiological range of concentration $(0.5-10 \mathrm{mM})$ inhibited both glucose uptake and flux through PFK-1 from exogenous glucose. However, the two steps differed in their sensitivity towards lactate. Indeed, inhibition of flux through PFK-1 was maximal at $5 \mathrm{mM}$ lactate, whereas inhibition of glucose uptake only started at $10 \mathrm{mM}$ lactate (Fig. 2). In hearts perfused with lactate, the concentrations of hexose 6-phosphates and citrate were increased, whereas the concentration of Fru-2,6-P2 was decreased, compared with hearts perfused with glucose alone (Table I). The increase in Fru-2,6-P2 concentration brought about by increasing the work load in glucose-perfused hearts was abolished in the presence of lactate. In all hearts perfused with lactate, the concentrations of Fru-2,6-P2 and citrate were inversely related (Fig. 3). The glycogen content was found to be increased by lactate in both work load conditions (Table I). Similar results were found with oxidizable substrates other than lactate. In hearts perfused with palmitate or $\beta$-hydroxybutyrate, glycogen concentration was $16.0 \pm 2.0$ and $13.5 \pm 2.0$ $\mu \mathrm{mol} / \mathrm{g}$, respectively, whereas Fru-2,6-P2 content was decreased to $0.7 \pm 0.1$ and $0.6 \pm 0.2 \mathrm{nmol} / \mathrm{g}$, respectively $(P<0.01$ versus hearts with glucose alone).

\section{Stimulation of glucose utilization by CPT-cAMP}

Addition of $0.1 \mathrm{mM} \mathrm{CPT-cAMP}$ to hearts perfused with glucose alone stimulated both hydraulic power and glycolysis from exogenous glucose in low load conditions. The stimulatory effect on hydraulic power occurred within the first minutes after the administration of CPT-cAMP, whereas the stimulation of glucose uptake was maximal only after $5 \mathrm{~min}$. At high load, however, the analogue did not further stimulate work and glucose metabolism. As expected (27), CPT-cAMP stimulated glycogen breakdown (Table I). To exclude that this glycogenolysis resulted from ischaemia brought about by the analogue, we checked that oxygen consumption increased from $7 \pm 1$ to $16 \pm 3 \mu \mathrm{mol} / \mathrm{min}$ per $\mathrm{g}$ after addition of CPT- 


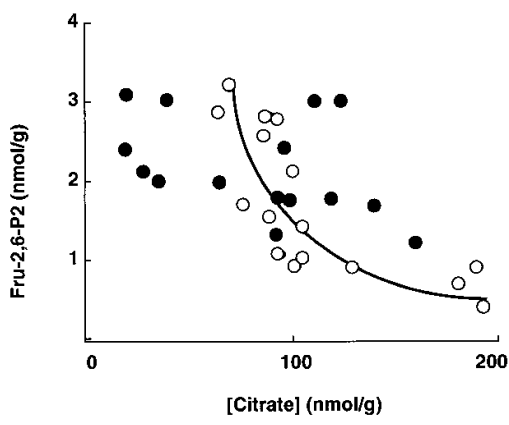
cant correlation found between both parameters in the absence of CPT-cAMP $(r=0.98, P<0.01)$. In the presence of the analogue, no significant correlation was found.

cAMP. Moreover, the fact that the hearts maintained a stable external work despite the exhaustion of their glycogen stores further excludes an ischemic insult. Hexose 6-phosphates were not affected by CPT-cAMP (Table I). The concentration of citrate was decreased significantly by the analogue (Table I), whereas Fru-2,6-P2 content changed in parallel with glycolysis from exogenous glucose (Table I and Fig. 1).

Addition of CPT-cAMP in the presence of an alternative substrate increased hydraulic power in low load conditions, as was the case for hearts perfused with glucose alone (Fig. 1). Remarkably, the increase of external work was matched with a similar stimulation of both glycolysis and glycogenolysis (Fig. 1 and Tables I and II), suggesting that the extra energy required was provided by glucose and glycogen, despite the presence of other oxidizable substrates. At high load, CPT-cAMP did not further increase the work developed, but completely relieved the inhibition of glycolytic flux by the alternative substrates (Fig. 1). In this case, glucose metabolism was clearly stimulated despite the presence of alternative substrates and despite any further increase in work.

The effects of the analogue were also tested in the presence of various concentrations of lactate. The analogue abolished the inhibition of glucose uptake and flux through PFK-1 from exogenous glucose at all concentrations of lactate tested (Fig. 2 ). The CPT-cAMP-induced glycogen breakdown was not affected by lactate (Table I). Citrate concentration was also decreased in the presence of CPT-cAMP. Interestingly, the decrease in Fru-2,6-P2 induced by lactate was relieved by CPTcAMP (Table I). Moreover, the inverse relationship between citrate and Fru-2,6-P2 concentrations that was observed in hearts perfused with lactate could not be evidenced when hearts were perfused with CPT-cAMP (Fig. 3).

As the glycogen stores were completely depleted in hearts perfused for $20 \mathrm{~min}$ with CPT-cAMP, a shorter perfusion period $(10 \mathrm{~min})$ was chosen to detect changes in metabolite concentrations during active glycogen breakdown. The concentration of hexose 6-phosphates was increased by up to $35 \%$ depending on the experimental groups. There was no significant change in Fru-1,6-P2 concentration, and the stimulatory effect of CPT-cAMP on Fru-2,6-P2 content was present in low load conditions (Table II).

\section{Relative contribution of exogenous glucose and glycogen to glycolysis}

All the rates reported in this section were calculated from the data reported in Fig. 1 and Table II. Under low load conditions, no significant loss of glycogen was measured and glucose was therefore the only source of carbohydrate for glycolysis $(\sim 0.6 \mu \mathrm{mol}$ glucose/min per $\mathrm{g})$. This rate was little affected by alternative oxidizable substrates (the $25 \%$ inhibition of PFK-1 flux by palmitate was not statistically significant), whereas it was greatly increased by CPT-cAMP. Under this condition, total glycolysis amounted to $\sim 2 \mu \mathrm{mol}$ glucose/min per $\mathrm{g}$ and the contribution of glucose and glycogen to total glycolysis was approximately equal.

Increasing the work load stimulated total glycolysis $(\sim 1.3$ $\mu \mathrm{mol} / \mathrm{min}$ per $\mathrm{g}$ ), with exogenous glucose and glycogen representing approximately two-thirds and one-third of the total rates, respectively. Addition of alternative substrates restored a glycolytic rate similar to that observed in low load conditions. Remarkably, the increase in glycogen content $(\sim 3 \mu \mathrm{mol} / \mathrm{g}$ in $10 \mathrm{~min}$ ) measured when lactate or palmitate was present accounted for $50-75 \%$ of the inhibition of glycolysis from glucose, thus indicating that exogenous glucose had been redirected toward glycogen. Finally, this effect of alternative substrates on both glycolysis and glycogen was completely suppressed by CPT-cAMP. In this condition, glycolysis from exogenous glucose averaged $1.1 \mu \mathrm{mol} / \mathrm{min}$ per $\mathrm{g}$ and glycogenolysis $0.5 \mu \mathrm{mol} / \mathrm{min}$ per $\mathrm{g}$.

Table II. Effect of CPT-cAMP on the Concentration of Glycogen, Hexose 6-P, Fru-1,6-P2, Citrate, and Fru-2,6-P2 in Hearts Perfused during 10 min in Two Work Load Conditions, with Glucose (glu) or Glucose and Lactate (glu/lac)

\begin{tabular}{|c|c|c|c|c|c|c|c|}
\hline Work load & Substrate & СРТ-cАMP & Glycogen & Hexose 6-P & Fru-1,6-P2 & Citrate & Fru-2,6-P2 \\
\hline & & & $\mu \mathrm{mol} / \mathrm{g}$ & & $\mathrm{nmol} / \mathrm{g}$ & & \\
\hline \multirow[t]{4}{*}{ Low load } & glu & - & $12.0 \pm 1.0$ & $71 \pm 6$ & $34 \pm 5$ & $34 \pm 6$ & $2.1 \pm 0.2$ \\
\hline & & + & $3.2 \pm 0.6^{*}$ & $81 \pm 5^{*}$ & $40 \pm 6$ & $36 \pm 5$ & $2.7 \pm 0.2 *$ \\
\hline & $\mathrm{glu} / \mathrm{lac}$ & - & $15.0 \pm 0.5^{\ddagger}$ & $122 \pm 10^{\ddagger}$ & $48 \pm 6^{\ddagger}$ & $113 \pm 10^{\ddagger}$ & $1.3 \pm 0.2^{\ddagger}$ \\
\hline & & + & $6.0 \pm 0.4^{* \neq}$ & $135 \pm 15^{\ddagger}$ & $53 \pm 7$ & $110 \pm 5^{\ddagger}$ & $1.8 \pm 0.2^{* \ddagger}$ \\
\hline \multirow[t]{4}{*}{ High load } & glu & - & $10.5 \pm 0.5$ & $73 \pm 3$ & $43 \pm 5$ & $22 \pm 3$ & $2.8 \pm 0.3^{\S}$ \\
\hline & & + & $1.0 \pm 0.3 * \S$ & $100 \pm 6^{*}$ & $26 \pm 3 *$ & $33 \pm 5^{*}$ & $3.0 \pm 0.2$ \\
\hline & glu/lac & - & $17.0 \pm 0.6^{\ddagger}$ & $160 \pm 10^{\ddagger}$ & $53 \pm 6$ & $125 \pm 5^{\ddagger}$ & $1.2 \pm 0.2^{\ddagger}$ \\
\hline & & + & $9.0 \pm 1.0^{* *}$ & $215 \pm 15^{* \neq}$ & $70 \pm 8$ & $153 \pm 8^{* \neq}$ & $1.5 \pm 0.1^{\ddagger}$ \\
\hline
\end{tabular}

Hearts were freeze-clamped after $10 \mathrm{~min}$ of perfusion in each condition. Hexose 6-P, hexose 6-phosphates. The values are the means \pm SEM for at least four different perfusions. $* P<0.05$ vs. corresponding value without CPT-cAMP. ${ }^{\ddagger} P<0.05$ vs. corresponding value in the same work load condition with glucose alone. ${ }^{\S} P<0.05$ vs. corresponding value at low load. 


\section{Molecular targets of CPT-cAMP}

From the results described above, it appears that the cAMP analogue relieved the inhibition exerted by lactate at the level of various regulatory steps in glucose metabolism, namely glucose uptake, flux through PFK-1, and glycogen breakdown. Such a stimulation of glycolytic flux was most probably matched with increased glucose oxidation and, therefore, with activation of PDH. Therefore, we measured the activity of PFK-2, phosphorylase, and PDH in each group.

PFK-2 activity. The stimulation of flux through PFK-1 by CPT-cAMP was related to an increased concentration of Fru2,6-P2 (Tables I and II). Moreover, the lactate-induced fall in Fru-2,6-P2 concentration was partly relieved by CPT-cAMP. This suggests a change in PFK-2 activity, either by decreasing the sensitivity to citrate or by increasing the $V_{\max }$. To test this hypothesis, we studied the effects of phosphorylation by PKA on the kinetic properties of purified bovine heart PFK-2 (Fig. 4). The results from at least three different experiments indicated that phosphorylation increased the $V_{\max }$ from 40 to 90 $\mathrm{mU} / \mathrm{mg}$ and decreased the $K_{\mathrm{m}}$ for Fru-6-P from 90 to $50 \mu \mathrm{M}$. Moreover, phosphorylation also increased the competitive $\left(K_{\mathrm{i}, \mathrm{c}}\right.$ from 20 to $\left.40 \mu \mathrm{M}\right)$ and the noncompetitive $\left(K_{\mathrm{i} \text { unc }}\right.$ from 70 to $110 \mu \mathrm{M}$ ) inhibition constants for citrate. These $K_{\mathrm{m}}$ and $K_{\mathrm{i}}$ values are in the physiological range of concentrations (Tables I and II) and changes in kinetic properties are thus expected to occur in hearts perfused with CPT-cAMP.

Phosphorylase activity. The activation of phosphorylase by cAMP is a well-known phenomenon (27). As shown in Fig. 5 , the activity of phosphorylase was not affected by lactate, but increased by threefold after addition of CPT-cAMP. Total phosphorylase activity was similar between groups and averaged about $10 \mathrm{U} / \mathrm{g}$.

PDH activity. Total PDH activity was the same in all groups and averaged $4.3 \mathrm{U} / \mathrm{g}$. Increasing the work load in hearts perfused with glucose alone increased the percentage of
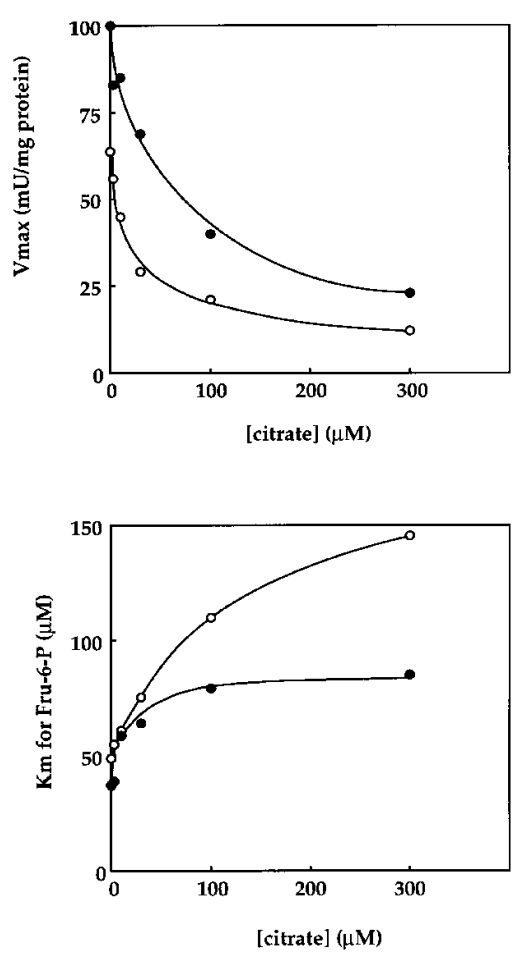

Figure 4. Inhibition of purified bovine PFK-2 by citrate before (open symbols) and after (closed symbols) phosphorylation by purified PKA. PFK-2 was measured in the presence of $5 \mathrm{mM}$ phosphate, $5 \mathrm{mM}$ $\mathrm{MgATP}$, and various concentrations of Fru6-P and citrate up to 10 times the respective $K_{\mathrm{m}}$ and $K_{\mathrm{i}}$ values. The results of one experiment are illustrated in this figure and mean values for $V_{\max }, K_{\mathrm{m}}$, and $K_{\mathrm{i}}$ are given in the text.
PDH from 29 to $96 \%$ (Fig. 6), whereas addition of lactate suppressed this stimulation at high load (Fig. 6). Similarly, addition of palmitate to hearts perfused at high load decreased the percentage of $\mathrm{PDH}$ in the active form down to $20 \pm 3 \%$; it is worth noting that this value was slightly lower than that observed with lactate $(29 \pm 3 \% ; P \leq 0.05)$. Addition of CPTcAMP fully activated the enzyme in all conditions (Fig. 6). The dose-response of $\mathrm{PDH}$ inactivation by various concentrations of lactate at high load (Fig. 2) further illustrates the difference in sensitivity of the key steps of glycolysis towards inhibition by lactate. PDH was the most sensitive step, followed by PFK-1, itself more sensitive than glucose uptake. These subtle differences in sensitivity were abolished by CPT-cAMP, whatever the concentrations of lactate used (Fig. 2). It is concluded that the stimulation of glucose utilization by cAMP resulted from a multisite stimulation involving glucose uptake, PFK-1, PDH, and glycogen breakdown.

\section{Discussion}

Many works have been conducted to investigate the inhibition of glucose metabolism by alternative substrates on one hand, and its stimulation by $\beta$-adrenergic receptors on the other hand. In this study, we mainly investigated how glucose metabolism is regulated when alternative substrates and cAMP are both present. Mainly, we show that cAMP reverses the inhibition of glycolysis exerted by alternative substrates. This involves a concerted stimulation of glucose transport, flux through PFK-1 (by stimulation of PFK-2), PDH activity, and glycogen breakdown (Fig. 7). We also showed that the metabolic effects of cAMP can be dissociated from its inotropic effect. Indeed, increasing the heart work did not suppress the inhibition of glycolysis by alternative fuels, whereas addition of CPT-cAMP in high load condition clearly induced a shift in heart metabolism without further enhancing the work.

We mainly investigated the effects of the cAMP analogue on hearts perfused with glucose and lactate as, after transformation into pyruvate, lactate and glucose share the same metabolic pathways (oxidation, carboxylation, or transamination). Thus, the fact that the inhibition of glycolysis by lactate is relieved by CPT-cAMP demonstrates that glucose becomes a preferred substrate. We will discuss the molecular mechanisms of the inhibition of glucose utilization by lactate and its reversal by CPT-cAMP.

Glucose uptake. Glucose uptake was affected by a relatively high concentration of lactate, possibly by a negative feed-back mechanism of hexose 6-phosphates on hexokinase

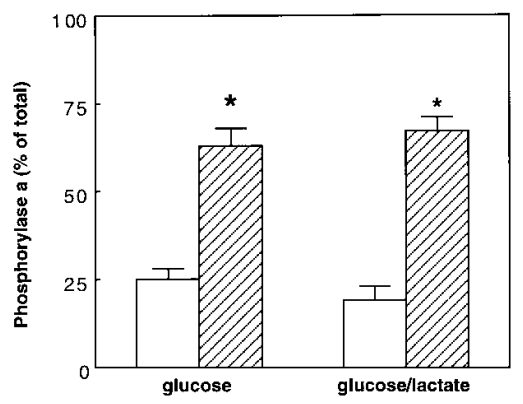

Figure 5. Percentage of phosphorylase in the active form in hearts perfused with glucose alone (left) or together with lactate (right), in the absence (open bars) or in the presence (hatched bars) of $0.1 \mathrm{mM}$ CPT-cAMP. $* P<0.05$ vs. same group without CPTcAMP. $n \geq 4$ in each group. 


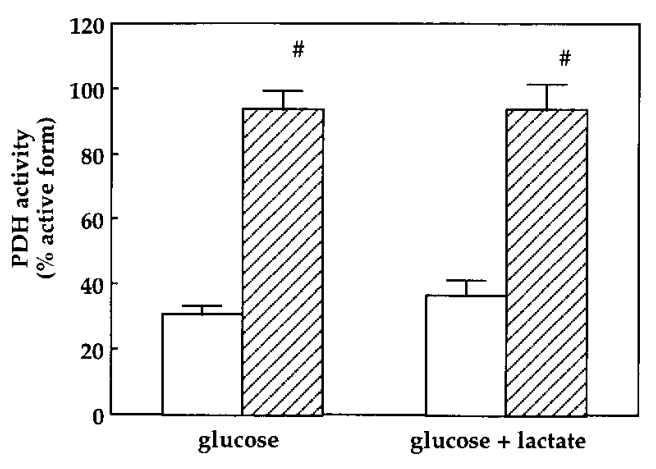

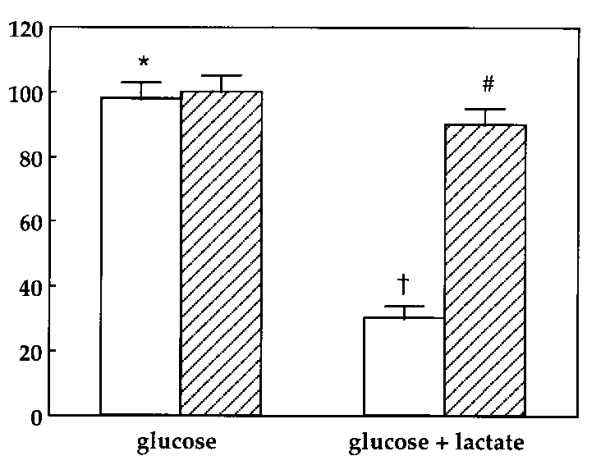

Figure 6. Percentage of PDH in the active form in hearts perfused with glucose alone or together with lactate, at either low load (left) or high load (right), in the absence (open bars) or in the presence (hatched bars) of $0.1 \mathrm{mM}$ CPT-cAMP. $* P<0.05$ vs. same group in low load condition; ${ }^{\dagger} P<$ 0.05 vs. same group without lactate; ${ }^{\#} P<0.05$ vs. same group without CPT-cAMP. $n \geq 4$ in each group.
(Table I). On the other hand, inhibition of PFK-1 flux was observed at concentrations of lactate lower than those needed to inhibit glucose uptake (Fig. 2). This difference in sensitivity, together with the lactate-induced increase in the concentration of glucose 6-phosphate, led to a reorientation of glucose metabolism toward glycogen synthesis. Indeed, the decrease in PFK-1 flux can account for the net increase in glycogen content (Figs. 1 and 2 and Table I). This accumulation of glycogen by lactate might be of metabolic importance since it confers some protection to hearts submitted to ischemic stress (28).

The effects of cAMP on glucose uptake have been deduced mainly from experiments with epinephrine, and conflicting

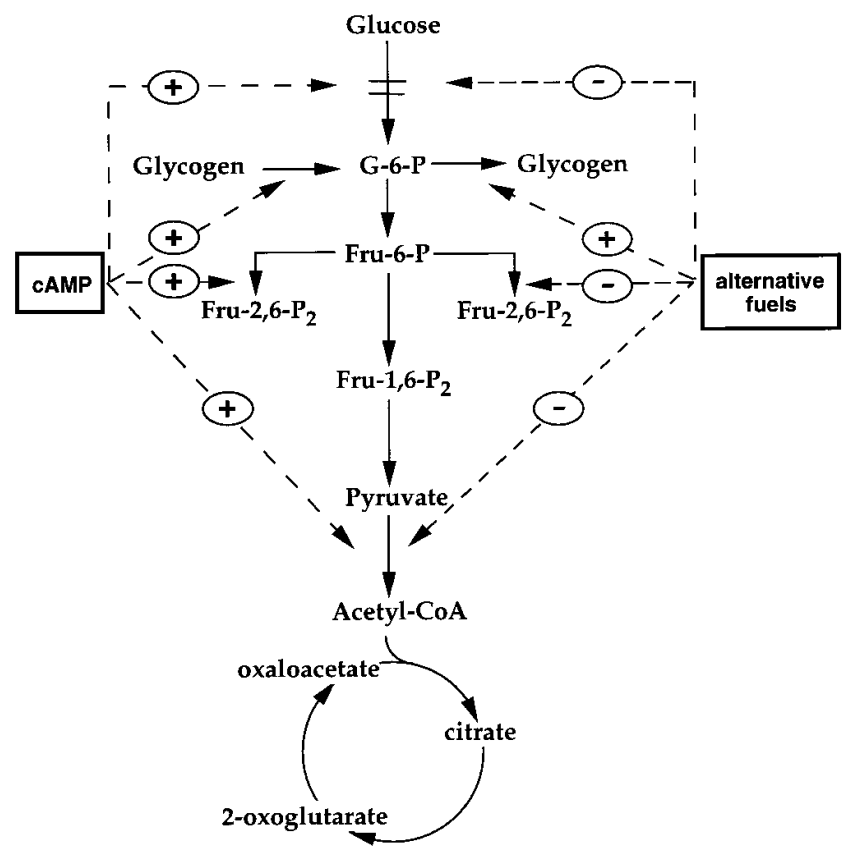

Figure 7. Inhibition of glucose utilization by alternative oxidizable substrates and its reversal by cAMP. Alternative substrates inhibit glucose metabolism through decreased glucose uptake, decreased production of Fru-2,6-P2, and decreased activity of PDH. These effects reroute glucose toward glycogen synthesis. On the contrary, cAMP increases myocardial glucose utilization through stimulation of glucose uptake, glycolytic flux, glycogen breakdown, and pyruvate oxidation. data have been reported. For Rattigan et al. (29), epinephrine stimulates the recruitment of GLUT-4 transporters through increased production of cAMP, whereas, for Fischer et al. (30), the stimulation of glucose transport by catecholamines is mediated through $\alpha$-adrenergic receptors and is cAMP independent. The latter data were obtained in nonbeating cardiomyocytes and could suggest that epinephrine controls glucose uptake by two different mechanisms: one cAMP-dependent and related to its inotropic effect, the other cAMP-independent mediated by $\alpha$-adrenergic receptors. Besides a potential effect of PKA on glucose transporters, cAMP may also stimulate glucose transport through increased calcium transients (6). In our working model, the inotropic effect of cAMP is clearly associated with a stimulation of glucose transport needed to encounter the increased metabolic demand at low load. At high load, however, CPT-cAMP did stimulate glucose uptake (Fig. 2) despite the absence of inotropic effect.

Glycogen metabolism. The addition of CPT-cAMP dramatically accelerated glycogen breakdown, due to an activation of phosphorylase (Fig. 5). This activation results from a stimulation of the glycogenolytic cascade by PKA and from the stimulation of phosphorylase kinase by increased calcium transients $(27,31)$.

Flux through PFK-1 and Fru-2,6-P2 concentration. Addition of lactate, within a physiological range of concentrations, led to a marked inhibition of PFK-1 flux. This inhibition probably results from both the fall in the concentration of Fru-2,6$\mathrm{P} 2$ and the increase in citrate content. Fru-2,6-P2 is indeed a stimulator, whereas citrate is an inhibitor of PFK-1. Citrate also increases the sensitivity of PFK-1 to inhibition by ATP (32). Moreover, heart PFK-2 is very sensitive to inhibition by citrate $(5,32)$. Therefore, citrate exerts a dual lock by inhibiting both PFK-1 and PFK-2, explaining that the concentrations of Fru-2,6-P2 and citrate were inversely related in hearts perfused without cAMP (Fig. 3). Whereas the inhibitory effect of citrate on heart PFK-1 has been described more than $30 \mathrm{yr}$ ago (3), the mechanism of transfer of citrate from the mitochondria to the cytosol in cardiac cells is not completely understood. Nevertheless, the cytosolic and mitochondrial concentrations of citrate are closely related under various conditions in the heart (20), the cytosolic concentration being approximately half that in the mitochondria (33). Therefore, the concentrations of citrate in the cytosol in our experiments (Tables I and II) are well in the range of concentrations that affect PFK-2 activity (Fig. 6). 
To explain a stimulation of PFK-1 flux by cAMP, one may consider two possibilities, i.e., the PKA-dependent phosphorylation of PFK-1 or PFK-2. PFK-1 from various mammalian tissues has been reported to be a substrate for several protein kinases, including PKA $(34,35)$. Whether phosphorylation induces changes in kinetic properties of PFK-1 is a matter of dispute $(36,37)$ and convincing evidence to consider PFK-1 phosphorylation as a major control mechanism in the heart is still lacking. This is not the case for heart PFK-2, which is activated after phosphorylation by PKA $(38,39)$. The present data confirm that phosphorylation changes the kinetic properties and favors the synthesis of Fru-2,6-P2 by decreasing the $K_{\mathrm{m}}$ for fructose-6-phosphate, and by increasing the $V_{\max }$. Moreover, we show here that phosphorylation renders PFK-2 less sensitive to citrate inhibition (Fig. 4). This mechanism explains the stimulation of flux through PFK-1 and the lack of correlation between Fru-2,6-P2 and citrate in hearts perfused with CPTcAMP (Fig. 3).

PDH activity. The activity of $\mathrm{PDH}$ is under tight control by effectors and by phosphorylation/dephosphorylation, the active form being dephosphorylated. PDH kinase, the inactivating enzyme, is stimulated by the reaction products, acetylCoA and NADH, whereas PDH phosphatase is stimulated by calcium $(24,25)$. The activation of PDH by increasing the work load (Fig. 6) probably results from an activation of PDH phosphatase by increased calcium transients. The activation of PDH by catecholamines (15) is transduced by $\beta$-agonist receptors (40), whose effects are mediated by cAMP. Our experiments indeed show that CPT-cAMP fully activated PDH. The exact mechanism leading to $\mathrm{PDH}$ activation by cAMP remains unknown, since PDH interconversion is not directly controlled by PKA, but is related to calcium transients. However, the mechanism may be more complex. Indeed, the work loadmediated stimulation of $\mathrm{PDH}$ was abolished in the presence of lactate, whereas this was not the case in the presence of CPTcAMP (Fig. 2). As increased calcium transients occur in both conditions, other mechanisms must be implicated to explain an inhibitory effect of lactate in one condition and not in the other.

$\mathrm{PDH}$ inactivation by lactate under high load conditions deserves some comments. Although it may seem counterintuitive, it is actually expected from the known control of PDH interconversion (41). Both products of lactate dehydrogenase have antagonistic effects on $\mathrm{PDH}$ interconversion: an increase in the NADH/NAD ratio favors inactivation by stimulating the inactivating $\mathrm{PDH}$ kinase, whereas an increase in pyruvate promotes $\mathrm{PDH}$ activation by inhibiting the inactivating $\mathrm{PDH}$ kinase. We suggest that $\mathrm{PDH}$ inactivation by lactate results from a difference in the time-course of the change in NADH/ NAD ratio compared with the change in pyruvate content. After the addition of lactate, a drastic increase in NADH/NAD ratio is expected to occur immediately, whereas the accumulation of pyruvate would be more progressive. In addition, relatively high concentrations of pyruvate are required to change the activation state of $\mathrm{PDH}$, whereas relatively minor changes in NADH/NAD ratio are able to control PDH kinase (41). It is worth noting that PDH inactivation by lactate was not complete and was most evident when PDH had been activated previously by increasing the work load.

A mechanism involving an increase in the NADH/NAD ratio as well as in the acetyl $\mathrm{CoA} /$ free $\mathrm{CoA}$ ratio has also been invoked to explain $\mathrm{PDH}$ inactivation by palmitate in the heart.
Therefore, $\mathrm{PDH}$ inactivation by palmitate and lactate share a common regulatory event, namely the increase in NADH/ NAD ratio. However, PDH inactivation by palmitate is expected to be more pronounced than after lactate addition, because two inhibitors are produced from palmitate (NADH and acetyl-CoA), whereas one inhibitor (NADH) and one activator (pyruvate) are formed from lactate. A slight difference in the extent of PDH inactivation was indeed observed (lactate, $29 \pm 3 \%$; palmitate, $20 \pm 3 \% ; P \leq 0.05$ ).

Finally, our data show that PDH was more sensitive to inhibition by lactate than flux though PFK-1, itself more sensitive than glucose uptake. These differences in sensitivity may explain the rerouting of glucose toward both glycogen synthesis (from the difference between glucose uptake and PFK-1 flux), and anaplerosis of the tricarboxylic acid cycle (from the difference between PFK-1 flux and PDH activity).

\section{Acknowledgments}

We are indebted to Mrs. V. O'Connor for expert secretarial assistance.

C. Depre was Research Assistant of the National Fund for Scientific Research (Belgium), J. Deprez is supported by the Fund for Scientific Research in Industry and Agriculture. This work was supported in part by the National Fund for Medical Research (Belgium), the D.G. Higher Education and Scientific Research-French Community of Belgium, and the Belgian Programme on Interuniversity Poles of Attraction initiated by the Prime Minister's office.

\section{References}

1. Shipp, J.C., L.H. Opie, and D.R. Challoner. 1961. Fatty acid and glucose metabolism in perfused rat heart. Nature. 189:1018-1019.

2. Randle, P.J., P.B. Garland, C.N. Hales, and E.A. Newsholme. 1963. The glucose/fatty acid cycle. Its role in insulin sensitivity and the metabolic disturbances of diabetes mellitus. Lancet. 1:785-789.

3. Garland, P.B., P.J. Randle, and E.A. Newsholme. 1963. Citrate as an intermediary in the inhibition of phosphofructokinase in rat heart muscle by fatty acids, ketone bodies, pyruvate, diabetes and starvation. Nature. 200:169-170.

4. Pogson, C.I., and P.J. Randle. 1966. The control of rat heart phosphofructokinase by citrate and other regulators. Biochem. J. 100:683-693.

5. Hue, L., L. Maisin, and M.H. Rider. 1988. Palmitate inhibits liver glycolysis. Involvement of fructose 2,6-bisphosphate in the glucose/fatty acid cycle. Biochem. J. 251:541-545.

6. Wheeler, T.J., R.D. Fell, and M.A. Hauck. 1994. Translocation of two glucose transporters in heart: effects of rotenone, uncouplers, workload, palmitate, insulin and anoxia. Biochim. Biophys. Acta. 1196:191-200.

7. Williamson, J.R. 1962. Effects of insulin and diet on the metabolism of $\mathrm{L}(+)$-lactate and glucose by the perfused rat heart. Biochem. J. 83:377-383.

8. Depre, C., K. Veitch, and L. Hue. 1993. Stimulation of glycogen synthesis by lactate in the isolated working rat heart. Acta Cardiol. 48:147-164.

9. Bristow, M.R., R. Cubbicciotti, and R. Ginsburg. 1982. Histamine-mediated adenylate cyclase activation in human myocardium. Mol. Pharmacol. 21: 671-679.

10. Neer, E.J., and D.E. Clapham. 1988. Roles of G-protein subunits in transmembrane signaling. Nature. 333:129-134.

11. Lopaschuk, G.D., M. Michalak, and E.L. Wandler. 1989. Prostaglandin $\mathrm{E}$ receptors in cardiac sarcolemma. Identification and coupling to adenylate cyclase. Circ. Res. 65:538-545.

12. Cramb, G., R. Banks, E.L. Rugg, and J.F. Aiton. 1987. Actions of atrial natriuretic peptide (ANP) on cyclic nucleotide concentrations and phosphatidylinositol turnover in ventricular myocytes. Biochem. Biophys. Res. Commun. 148:962-970.

13. Depre, C., and L. Hue. 1994. Cyclic GMP in the perfused rat heart. FEBS Lett. 345:241-245.

14. Williamson, J.R. 1964. Metabolic effects of epinephrine in the isolated, perfused rat heart. J. Biol. Chem. 239:2721-2729.

15. Collins-Nakai, R., D. Noseworthy, and G.D. Lopaschuk. 1994. Epinephrine increases ATP production in hearts by preferentially increasing glucose metabolism. Am. J. Physiol. 267:H1862-H1871.

16. Depre, C., M.H. Rider, K. Veitch, and L. Hue. 1993. Role of fructose 2,6-bisphosphate in the control of heart glycolysis. J. Biol. Chem. 268:1327413279. 
17. Taegtmeyer, H., R. Hems, and H.A. Krebs. 1980. Utilization of energyproviding substrates in the working rat heart. Biochem. J. 186:701-711.

18. Bontemps, F., L. Hue, and H.G. Hers. 1978. Phosphorylation in isolated rat hepatocytes. Biochem. J. 174:603-611.

19. Hue, L., and H.G. Hers. 1974. On the use of $\left[{ }^{3} \mathrm{H},{ }^{14} \mathrm{C}\right]$ glucose in the study of the so-called "futile cycles" in the liver and muscle. Biochem. Biophys. Res. Commun. 58:532-539.

20. Neely, J.R., R.M. Denton, P.J. England, and P.J. Randle. 1972. The effects of increased heart work on the tricarboxylate cycle and its interactions with glycolysis in the perfused rat heart. Biochem. J. 128:147-152.

21. Bergmeyer, H.U. 1984. Methods of Enzymatic Analysis. 3rd ed. VCH, Weinheim.

22. Hue, L., F. Bontemps, and H.G. Hers. 1975. The effect of glucose and potassium ions on the interconversion of the two forms of glycogen phosphorylase and of glycogen synthetase in isolated rat liver preparations. Biochem. J. 152:105-114

23. Dopere, F., F. Vanstapel, and W. Stalmans. 1980. Glycogen-synthase phosphatase in rat liver. Two protein components and their requirement for the activation of different types of substrate. Eur. J. Biochem. 104:137-146.

24. McCormack, J.G., and R.M. Denton. 1980. Role of calcium ions in the regulation of intramitochondrial metabolism. Biochem. J. 190:95-105.

25. McCormack, J.G., N.J. Edgell, and R.M. Denton. 1982. Studies on the interaction of $\mathrm{Ca}^{2+}$ and pyruvate in the regulation of rat heart pyruvate dehydrogenase activity. Biochem. J. 202:419-427.

26. Deprez, J., D. Vertommen, D.R. Alessi, L. Hue, and M.H. Rider. 1997. Phosphorylation and activation of heart 6-phosphofructo-2-kinase by protein kinase B and other protein kinases of the insulin signaling cascades. J. Biol. Chem. 272:17269-17275.

27. Dobson, J.G., J. Ross, and S.E. Mayer. 1976. The role of cyclic adenosine $3^{\prime}, 5^{\prime}$-monophosphate and calcium in the regulation of contractility and glycogen phosphorylase activity in guinea pig papillary muscle. Circ. Res. 39:388-395.

28. Depre, C., and L. Hue. 1997. Inhibition of glycogenolysis by a glucose analogue in the working rat heart. J. Mol. Cell. Cardiol. 29:2253-2259.

29. Rattigan, S., G.J. Appleby, and M.G. Clark. 1991. Insulin-like action of catecholamines and $\mathrm{Ca}^{2+}$ to stimulate glucose transport and GLUT4 translocation in perfused rat heart. Biochim. Biophys. Acta. 1094:217-223.

30. Fischer, Y., J. Thomas, G.D. Holman, H. Rose, and H. Kammermeier.
1996. Contraction-independent effects of catecholamines on glucose transport in isolated rat cardiomyocytes. Am. J. Physiol. 39:C1204-C1210.

31. Morgan, H.E., and A. Parmeggiani. 1964. Regulation of glycogenolysis in muscle. II. Control of glycogen phosphorylase reaction in isolated perfused heart. J. Biol. Chem. 239:2435-2439.

32. Hue, L., and M.H. Rider. 1987. Role of fructose 2,6-bisphosphate in the control of glycolysis in mammalian tissues. Biochem. J. 245:313-324.

33. Wiesner, R.J., U. Kreutzer, P. Rosen, and M.K. Grieshaber. 1988. Subcellular distribution of malate-aspartate cycle intermediates during normoxia and anoxia in the heart. Biochim. Biophys. Acta. 36:114-123.

34. Clark, M.G., and G.S. Patten. 1981. Epinephrine activation of phosphofructokinase in perfused rat heart independent of changes in effector concentrations. J. Biol. Chem. 256:27-30.

35. Söling, H.D., and I.A. Brand. 1981. Covalent modification of phosphofructokinase by phosphorylation-dephosphorylation. Curr. Top. Cell. Regul. 20: $107-138$.

36. Kitajima, S., R. Sakakibara, and K. Uyeda. 1983. Significance of phosphorylation of phosphofructokinase. J. Biol. Chem. 258:13292-13298.

37. Hofer, H.W. 1985. Phosphorylation of phosphofructokinase. The possible role of covalent modification in the regulation of glycolysis. In Regulation of Carbohydrate Metabolism. Vol. I. R. Beitner, editor. CRC Press, Boca Raton. 105-141.

38. Kitamura, K., K. Kangawa, H. Matsuo, and K. Uyeda. 1988. Phosphorylation of myocardial fructose 6-phosphate, 2-kinase:fructose-2,6-bisphosphatase by cyclic AMP-dependent protein kinase and protein C. Activation by phosphorylation and amino acid sequences of the phosphorylation sites. J. Biol. Chem. 263:16796-16801.

39. Rider, M.H., J. Van Damme, D. Vertommen, A. Michel, J. Vandekerckhove, and L. Hue. 1992. Evidence for new phosphorylation sites for protein kinase $\mathrm{C}$ and cyclic AMP-dependent protein kinase in bovine heart 6-phosphofructo-2-kinase/fructose-2,6-bisphosphatase. FEBS Lett. 310:139-142.

40. Hiraoka, T., M. DeBuysere, and M.E. Olson. 1980. Studies on the effects of $\beta$-adrenergic agonists on the regulation of pyruvate dehydrogenase in the perfused rat heart. J. Biol. Chem. 255:7604-7609.

41. Kerbey, A.L., P.J. Randle, R.H. Cooper, S. Whitehouse, H.T. Pask, and R.M. Denton. 1976. Regulation of pyruvate dehydrogenase in rat heart. Biochem. J. 154:327-348. 